Journal of

Clinical and Anatomic Pathology

\title{
Evaluation of Liver Fibrosis in Cirrhotic Patients: Comparison between Elastoso- nography and Fibroscan
}

\section{Silvia Roma ${ }^{1}$, Cristina Pace ${ }^{1}$, Andrea Amico ${ }^{1}$, Ilaria Lenci ${ }^{2}$, Simona Francioso ${ }^{2}$, Carlo Chiaramonte ${ }^{3}$,- Fabrizio Chegai ${ }^{1}$, Antonio Orlacchio ${ }^{1, *}$}

${ }^{1}$ Department of Diagnostic and Interventional Radiology, University Hospital Tor Vergata, Viale Oxford 81, 00133 Rome, Italy ${ }^{2}$ Liver Unit - University Hospital Tor Vergata, Viale Oxford 81, 00133 Rome, Italy

${ }^{3}$ Statistic Unit - University Hospital Tor Vergata,Viale Oxford 81, 00133 Rome, Italy

*Corresponding author: Antonio Orlacchio, MD, Department of Diagnostic and Interventional Radiology, University Hospital Tor Vergata, Viale Oxford 81, 00133 Rome, Italy, E-mail address: aorlacchio@uniroma2.it; Tel number: +390620902400 Fax: +390620902404

Received Date: June 19, 2019 Accepted Date: July 20, 2019 Published Date: July 22, 2019

Citation: Silvia Roma (2019) Evaluation of Liver Fibrosis in Cirrhotic Patients: Comparison between Elastosonography and Fibroscan. J Clin Anat Pathol 4: 1-8.

\begin{abstract}
To date, there are still no clear data on the validity of Shear Waves Elastography (SWE) techniques in assessing liver fibrosis. Our study aimed to compare the stiffness liver values obtained with SWE techniques with Transient Elastography (TE) and to identify the threshold values for the different degrees of fibrosis.

We enrolled 11 healthy subjects and 48 patients with chronic liver disease. They all performed TE, 33 patients and healthy subjects were evaluated withPoint SWE and 15 patients with 2D-SWE. The differences between techniques and TE, evaluated with student's t-test, were not statistically significant ( $p>0,05$ ). The cut-offs of SWE technique were for $F \geq 26,2 \mathrm{kPa}$; $\mathrm{F} \geq 37,65 \mathrm{kPa} ; \mathrm{F}=413 \mathrm{kPa}$.
\end{abstract}

SWE techniques have good potential in assessing liver fibrosis; moreover, being elastosonography quick and easy to perform, it could be integrated into the clinical practice of the ultrasound examination.

Keywords: Liver fibrosis, Shear-wave elastography, Transient elastography, Cirrhosis, Chronic liver disease

(C)2019 The Authors. Published by the JScholar under the terms of the Creative Commons Attribution License http://creativecommons.org/licenses/ by/3.0/, which permits unrestricted use, provided the original author and source are credited. 


\section{Introduction}

Chronic liver diseases are an important public health problem, with an incidence in Europe of 5,82\% [1]. They have multiple causes, many of which are increasing in prevalence. The final common pathway of chronic liver disease is tissue destruction and attempted regeneration, a pathway that triggers fibrosis and eventual cirrhosis. Assessment of fibrosis is important not only for diagnosis but also for management, prognostic evaluation, and follow-up of patients with chronic liver disease.

For years, liver biopsy has been considered the standard reference in the staging of hepatic fibrosis. However, approximately $25 \%$ of patients experience pain during the invasive procedure, and $0.3 \%-0.6 \%$ of patients experience severe complications, such as bleeding and even death [2].

To replace liver biopsy, non-invasive approaches based on serologic methods, which however can be influenced by factors not related to hepatic function, and on imaging techniques were developed for liver fibrosis estimation.

To date, Fibroscan, introduced in 2003 as the first imaging technique that uses transient elastography (TE) able to measure the degree of hepatic fibrosis in a non-invasive way, is a reference point for hepatologists to classify patients with chronic liver disease [3].

Because hepatic fibrosis increases the stiffness of the hepatic parenchyma due to an increase in the extracellular matrix, elastosonography can be used to assess fibrosis degree. This method allows analyzing tissue behavior when it is subjected to mechanical stress using ultrasound.

There are two basic methods of elastosonography: the strain method (SE) and the shear-waves method (SWE). StrainElastographyevaluates the strain of a tissue induced by an external stimulus [4]. Shear-Wave based techniques, instead, measure the speed of Shear-waves in tissues. The main difference between these techniques is that the speed of the shear-waves, being linked to the stiffness, can be measured and converted into $\mathrm{KPa}$, while the strain elastography provides only a relative estimate [5]. Shear-Wave based techniques include TE, point SWE, and 2D-, 3D- SWE.TE evaluates the speed of a Shear Wave generated by an automated movement of a piston. Point SWE measures the average speed of Shear Wave propagation, generated using acoustic radiation force, from one lateral boundary of a measurable ROI to the opposite lateral boundary of the ROI; in 2D-, 3D- SWE the method described for point SWE is used to create even a quantitative image. The arrival time of the shear waves is evaluated in several lateral positions, this allows to create a large 2D-SWE ROI image, which is displayed in color or grayscale. In both techniques, ultrasound imaging is used to guide the placement of the ROI [6].

Nowadays almost all the manufacturers of ultrasounds have activated SWE techniques on their most recent machines, but the lack of sufficient data for these last machines, due to their recent introduction, as well as the considerable decrease in the number of liver biopsies in clinical practice, has raised some pertinent issues, in particular the possibility of using the stiffness values adopted for the staging of chronic hepatic diseases with TE also with other SWE techniques. The first studies have documented a good potential of the SWE techniques in the evaluation of liver fibrosis, but they warn about using the same thresholds of TE for different degrees of fibrosis because they found modestly lower values with SWE techniques compared to TE for the same degree of fibrosis.

Our study aimed to compare the stiffness values of the liver obtained with two different shear wave techniques with the corresponding values obtained by TE in the same patients and to identify the threshold values for the different degrees of fibrosis.

\section{Materials and Methods}

\section{Patients}

This is a prospective study. We enrolled 11 volunteer healthy subjects and 48 patients with chronic liver disease (patients and healthy subjects characteristics are shown in table 1) followed at the Hepatology Department. Patients were selected after evaluation of the histological data, to have homogeneity of the different degrees of fibrosis. Healthy subjects were medical staff members (nurses, residents and medical doctors), who are regularly tested and screened for HCV/HBV. They didn't have a history of liver disease and presented a normal aspect of the liver on ultrasound examination. The study protocol was approved by the Ethics Committee of our Institute and all the patients gave their written informed consent to the study. The exclusion criteria were hepatic transplantation, biliary obstruction, compromised clinical conditions, pregnancy. Thirty-nine $(81,25 \%)$ patients received a diagnosis of chronic hepatitis caused by 


\begin{tabular}{|l|l|l|l|l|}
\hline & \multicolumn{2}{|c|}{ PATIENTS } & \multicolumn{2}{c|}{ HEALTHY PARTECIPANTS } \\
\hline & $\begin{array}{l}\text { Median (IQR) or Abso- } \\
\text { lute Count } \\
36\end{array}$ & Range & $\begin{array}{l}\text { Median (IQR) or } \\
\text { Absolute Count } \\
5\end{array}$ & Range \\
MALE & 12 & $39-83$ & 6 & $50(15)$ \\
\hline FEMALE & $61(18)$ & $18-36$ & $22,9(5,2)$ & $35-62$ \\
AGE (YEARS) & $24,2(5)$ & $11,1-282$ & $19(9,6)$ & $12-27,5$ \\
\hline BMI (KG/M2) & $32(18,8)$ & $16-190$ & $17(6,7)$ & $10,3-21$ \\
ALT (U/L) & $35,5(32,9)$ & $12-221$ & $16(5)$ & $10-25$ \\
\hline AST (U/L) & $45(50,6)$ & $64-138$ & $73(31,3)$ & $45-89$ \\
GGT(U/L) & $95(37,2)$ & $108-328$ & $268(42)$ & $230-335$ \\
\hline ALKALINE PHOSPHATASE (U/L) & $211(88)$ & &
\end{tabular}

Table 1:Patients and Healthy Partecipants characteristics.Data are reported as medians and ranges or as absolute count. BMI: body mass index. ALT: alanine transaminase. AST: aspartate transaminase. GGT: gamma gluthamyl synthetase.

hepatitis $\mathrm{C}$ virus(HCV), 4 (8,33\%) patients had chronic liver disease related to hepatitis B virus (HBV), 1 (2,08\%) patient presented alcoholic liver disease and $4(8,33 \%)$ patients had autoimmune hepatitis.

Everyone performed TE (Fibroscan FS502, Echosens, Paris France) and a general abdomen US examination, 33 patients $(68,75 \%)$ and all healthy subjects were evaluated, using a convex probe C1-8, with Point Shear Wave Speed (SWS) measurements (QElaXto, MyLab Eight EXP, Esaote, Genova, Italia),15patients (31,25\%)underwent SWS imaging (Aplio i800, Canon Medical Systems Europe B.V., Zoetermeer, The Netherlands) with convex probe i8CX1.

\section{TE and SWE elastography examination}

The two procedures were performed on different days, within a maximum distance of 30 days from each other. TE was performed on a patient lying supine with the right arm raised above the head. The operator placed the tip of the probe on the skin between $9^{\circ}$ and $11^{\circ}$ intercostal space and, aided by time-motion image, he identified a hepatic portion at least 6 $\mathrm{cm}$ deep and free of large vascular structures. Then he pressed the probe button and started the evaluation. Ten measurements were made for each patient.

The SWE elastography examination was performed on fasting, in the supine position with the right arm carried behind the head. The ultrasound probe was placed perpendic- ular to the skin and the region of interest (ROI) was positioned between 2-6 $\mathrm{cm}$ from the skin and at least one centimeter from the liver capsule, avoiding the large blood vessels and fibrotic shoots. A total of 10 measurements were made for each one, 8 intercostal measurements on the right hepatic lobe with the probe parallel to the intercostal space and 2 measurements under xiphoid on the left lobe. We performed these two measurements on the left hepatic lobe to check if they can be reliable in the assessment of the degree of fibrosis or if, in analogy to the assessment with Fibroscan, the cardiac movements can influence the acquired data. During the measurements, no pressure was applied to the liver and the patient was asked to stop breathing for a few seconds to reduce movement artifacts. The examinations were performed by one expert operator who was blind to the Fibroscan data. The evaluation time was an average of 10 minutes per patient. Reliable SWE measurements were obtained in all patients.

\section{Statistical analysis}

The stiffness values were expressed in $\mathrm{kPa}$ in all techniques used. To perform the statistical analysis the data were expressed as mean and standard deviation (SD). The share waves techniques and fibro can be compared by Student's t-test.

To evaluate the cut-off points for fibrosis and the accuracy of SWE techniques in discriminating between the different stages of fibrosis, the receiver-operating characteristic (ROC) curves were performed, based on histological data. For the ROC 
curves analysis, the area under the curve (AUC), along with 95\% confidence intervals (CI), the optimal cut off value, sensitivity, and specificity were computed. Youden's index was used to define optimal cut off values. Statistical significance was set at $P<0.05$. The statistical p-value was 2-sided. All statistical analyses were performed with IBM SPSS Statistics software (release 15; IBM, Armonk, NY, USA).

\section{Results}

Thirty-three patients were evaluated with Point SWEand TE, the stiffness mean values were respectively $12,74 \mathrm{kPa}$ $\pm 9,71 \mathrm{SD}$ and $12,78 \mathrm{kPa} \pm 15,43 \mathrm{SD}$ (Figure 1). These parameters were evaluated through a Student's $t$ distribution with 32 degrees of freedom. The difference between the two groups was not statistically significant $(p>0,05)$. Fifteen patients were instead evaluated with $2 \mathrm{D}-\mathrm{SWE}$ and TE, the stiffness means values were respectively $10,77 \mathrm{kPa} \pm 8,16 \mathrm{SD}$ and $10,88 \mathrm{kPa} \pm 5,04 \mathrm{SD}$ (Figure 2) and also in this case t-test showed no statistically significant difference between the two groups ( $p>0,05)$.

We also calculated in SWE techniques the stiffness mean value by eliminating the measurements performed on left hepatic lobe: for Point, SWEit was $12,40 \mathrm{kPa} \pm 9,54 \mathrm{SD}$ and for 2D-SWEwas $10,49 \pm 7,44$. Also in these cases, the differences with TE were not statistically significant $(p>0,05)$. The stiffness mean values of 11 healthy subjects, evaluated with Point

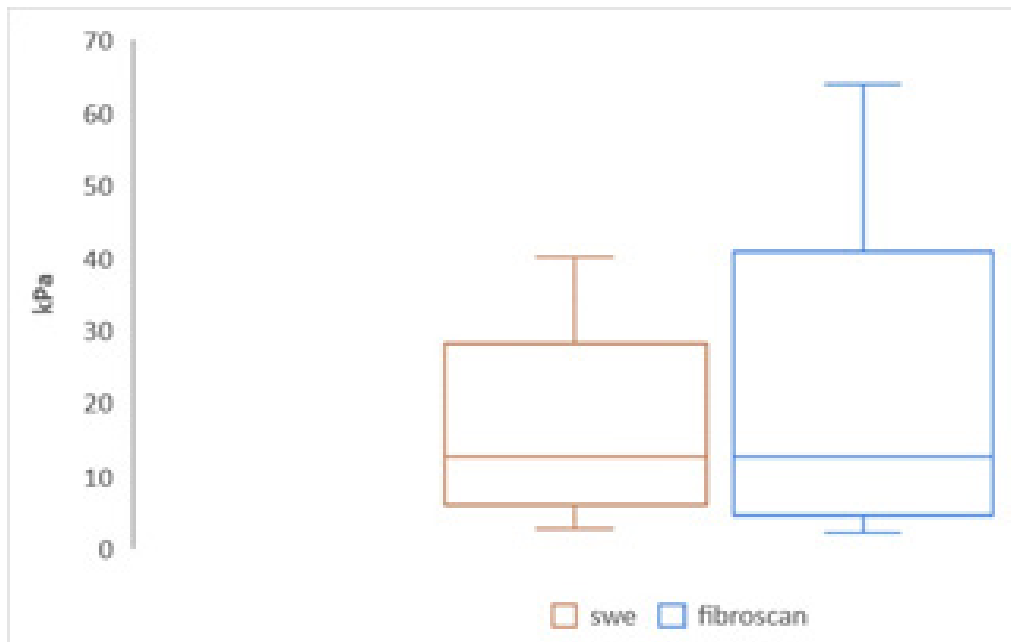

Figure 1. Box plots of liver stiffness values obtained with Point SWS measurements and TEin comparison. The top and the bottom of the boxes are the upper and lower limits of the $95 \%$ confidence interval. The line through the middle of each box represents the mean value.

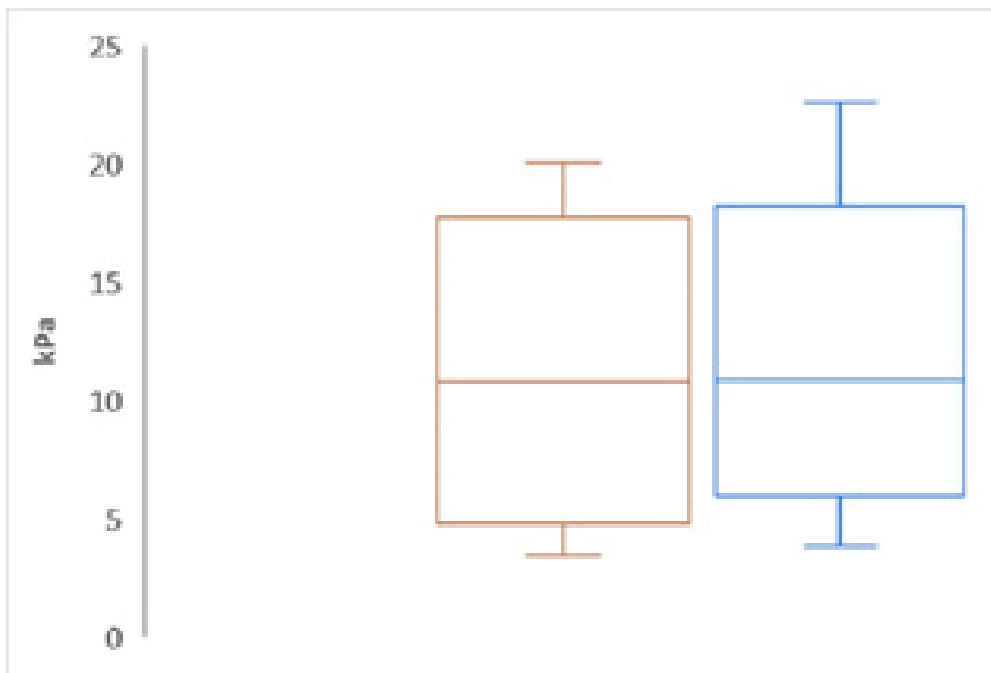

swe $\square$ fibroscan

Figure 2. Box plots of liver stiffness values obtained with SWS imaging and TE. The top and the bottom of the boxes are the upper and lower limits of the $95 \%$ confidence interval. The line through the middle of each box represents the mean value. 
SWEand TE, were respectively 4,72 $\mathrm{kPa} \pm 1,22 \mathrm{SD}$ and 4,91 $\mathrm{kPa} \pm 1,35 \mathrm{SD}$ and no statistically significant differences were observed. In this case, the stiffness means value by eliminating the measurements performed on the left hepatic lobe was 4,46 $\mathrm{kPa} \pm 1,28 \mathrm{SD}$

The cut-offs for SWE technique, calculated using ROC curves based on histological data as the reference gold standard, were for $\mathrm{F} \geq 26,2 \mathrm{kPa} ; \mathrm{F} \geq 37,65 \mathrm{kPa} ; \mathrm{F}=413 \mathrm{kPa}$. The evaluation of the area under the curve showed that SWE technique presented an accuracy of $85 \%$ in discriminating a moderate fibrosis (F2), of $74 \%$ in discriminating severe fibrosis (F3) and of 93\% in discriminating cirrhosis (F4) (ROC curves characteristics are shown in table 2 and Figure 3a-c).

\begin{tabular}{|l|l|l|l|}
\hline & $\mathrm{F} \geq 2$ & $\mathrm{~F} \geq 3$ & $\mathrm{~F}=4$ \\
\hline Cut off $(\mathrm{kPa})$ & 6,2 & 7,65 & 13 \\
\hline Sensitivity (\%) & 80 & 86 & 100 \\
\hline Specificity (\%) & 82 & 68 & 79 \\
\hline AUROC (95\% CI) & $0,85(0,73-0,98)$ & $0,74(0,57-0,91)$ & $0,93(0,83-1)$ \\
\hline
\end{tabular}

Table 2: Operative characteristics of shear-wave elastography in predicting fibrosis stages

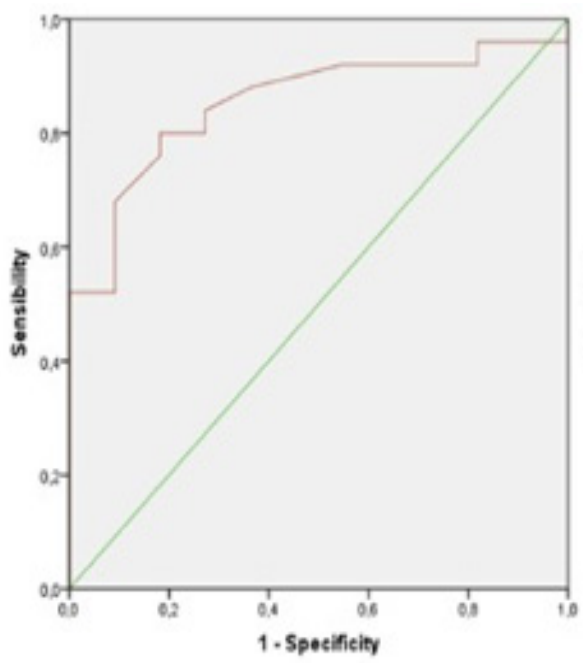

a

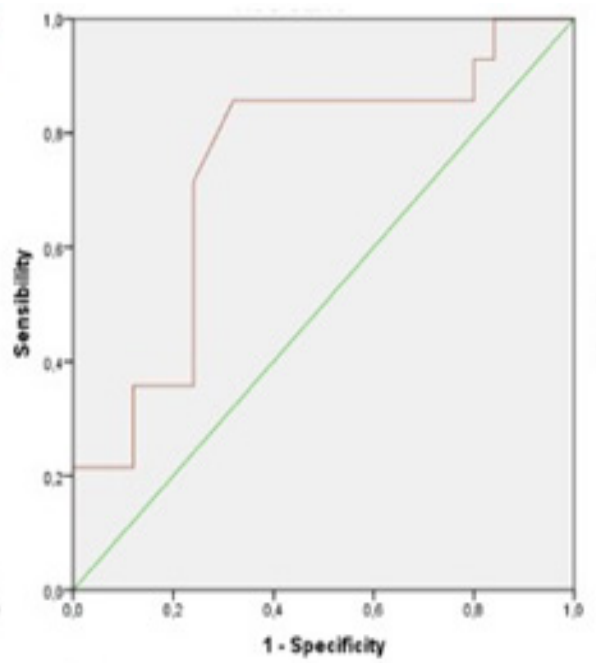

b

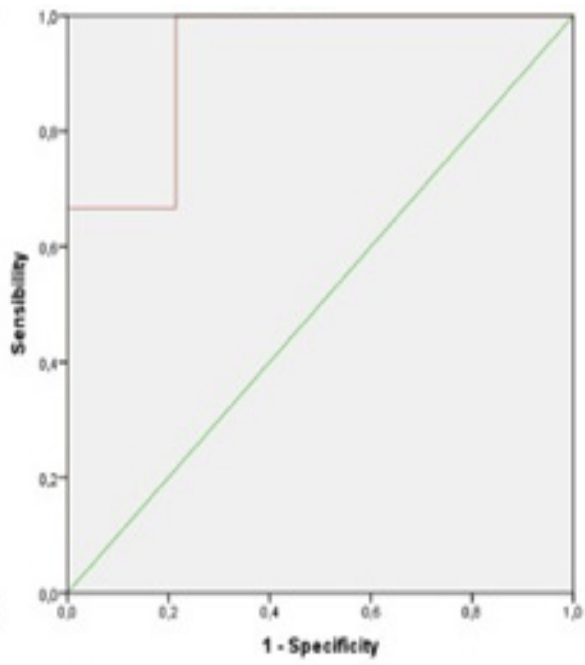

c

Figure 3.a) Receiver operating characteristic (ROC) curve for shear wave elastography in patients with fibrosis F2 vs F0; b) Receiver operating characteristic (ROC) curve for shear wave elastography in patients with fibrosis F3 vs F2; c) Receiver operating characteristic (ROC) curve for shear wave elastography for patients with fibrosis F4 vs F3.

\section{Discussion}

Fibrosis is a process characterized by an abnormal increase in the deposition of collagen and other components of the extracellular matrix. There are several systems of the staging of liver fibrosis based on histopathological data, one of the most used is the METAVIR score [7]. This system consists of four stages: F0 indicates no fibrosis, F1 is mild fibrosis characterized by fibrous portal expansion, F2 is moderate fibrosis with few bridges or septa, F3 is severe fibrosis with numerous bridges or septa and F4 is cirrhosis [8].

Liver biopsy is the most specific test to assess the nature and severity of the chronic liver disease, but it is an invasive procedure, not free from possible complications so it can not be used to monitor the progression of the disease or to evaluate the effect of therapy on the fibrotic process. Also, the biopsy sample represents 1 / 50,000 of the total liver mass while fibrosis is a heterogeneous process [9].

For these reasons, to date, the number of liver biopsies to evaluate liver stiffness degree is greatly reduced in favor of non-invasive techniques as Fibroscan. However Fibroscan has some limitations, it does not allow a simultaneous ultrasound evaluation of the liver, it has a limited liver scanning and it is contraindicated in patients with obesity or ascites [9].

In elastosonography, the possibility of performing a simultaneous ultrasound evaluation allows a wider sampling of the liver parenchyma and, being fibrosis a heterogeneous process, this could provide a more correct data of the liver fibrosis degree. 
As already mentioned, elastography is composed of two basic methods: Strain Elastography and Shear Wave Elastography. The first is a relative indicator of stiffness, that changes according to the degree of compression, besides strain imaging is essentially qualitative, for its quantification requires a comparison with a reference tissue [4]. Shear-Wave based techniques measure the speed of Shear-waves in tissues. The Share-waves can be generated by an external push (transient elastography) or by an ultrasound pulse that allows a single measurement (point shear wave speed measurement) or an image (shear wave speed imaging) $[5]$.

In our study, we evaluated the concordance between TE and the other SWE technique obtained with two US machines. Results of our study showed that there was no statistically significant difference between the stiffness values of the liver obtained with the different techniques. So we could assert that the two values distributions were overlapping and that any variation was due to chance.

Piscaglia et al. [3] in their study showed stiffness values obtained with SWE techniques lower than those obtained with TE; also in our case, the SWE stiffness values appear slightly lower but very close to the values obtained with the TE, without evidence of a statistically significant difference.

A further slight reduction of the stiffness values obtained with the SWE technique is appreciated by eliminating the two measurements carried out on the left hepatic lobe but, even in this case, a statistically significant difference with TE was not observed.

Also, a previous study found no statistically significant difference in tissue mean elasticity values between the right and left lobe of the liver in a study on 52 NASH patients [11]. Toshima et al. [12], instead, found that the values obtained on the right hepatic lobe were significantly lower than those obtained on the left hepatic lobe. This could be due to the anatomical position of the left hepatic lobe, being between diaphragm, aorta, and stomach, it could be influenced by the respiratory movement, by the pulsation of the aorta and by the presence of food in the stomach. Furthermore, the different thickness of the skin in the right intercostal space and in the region under xiphoid could influence the measurement.

We can hypothesize that in our study, the evaluation of patients on fasting and with stopped breath may have reduced the difference between measurements on the right hepatic lobe and the left hepatic lobe.

Several studies have highlighted that the stiffness thresholds established for Fibroscan for the classification of the different stages of liver fibrosis can not be adopted with the other SWE techniques $[3,10,13]$. Fraquelli et al. [10] in their study on 186 patients with chronic liver diseases showed slightly lower stiffness thresholds evaluated with Philips ElastoPQ technology compared to those of Fibroscan for the same grade of fibrosis histologically evaluated, as well as Ferraioli et al. 2014 [14] in a study on 92 healthy subjects reported lower point shear-wave elastography cut-off values for all stages of liver fibrosis as compared to Fibroscan values. Also in our study, we found the stiffness threshold slightly lower than those of Fibroscan, especially in the lower stages of fibrosis.

Mancini et al. [15] showed a cut off 5,62 kPa between F0-F1and F2-F4, this value was close to the cutoff of $6.2 \mathrm{kDa}$ reported by Chong Hyun Suh et al. [16] in their study.

As for Fibroscan, even in other SWE methods, an overlap of stiffness values between adjacent fibrosis stages is observed, particularly in the lower stages [5]. SWE techniques show a diagnostic accuracy similar to TE in the evaluation of cirrhosis $[17,18]$. This can be clinically relevant because, according to the American Association for the study of liver disease, among patients with hepatitis $\mathrm{C}$ genotype 1 infection, only those with moderate-severe fibrosis should be treated [19, 20]. Mancini et al. [15] in their study suggested that biopsy should be done in those patients with chronic liver disease who have SWE values below $7.04 \mathrm{kPa}$ to identify patients with F2 fibrosis.

There were some limitations in this study including the single-Center design and small study population, which may have influenced the results of the study. The ultrasound machines with SWE technique were available to us only for 15 days so that more patients could not be enrolled. However a total of 118 assessments were performed and patients were enrolled after evaluation of histological data to have homogeneity of the different degrees of fibrosis; moreover, we think that the evaluation of 11 healthy subjects can improve the results of the work and partially the limit of the small study population.

In conclusion, ours is an attempt to identify the threshold values for different degrees of liver fibrosis with SWE techniques. Our experience confirms that the SWE techniques have 
good potential in assessing liver fibrosis; moreover, as the elastosonography is quick and easy to perform, it could be integrated into the clinical practice of the ultrasound examination for the evaluation of liver stiffness degree without a significative increase of time of the exam. However, we believe that further studies on elastosonography techniques are applicable to identify more accurate threshold values for the different stages of liver fibrosis.

\section{References}

1. Blachier M, Leleu H, Peck-Radosavljevic M, Valla DC, Roudot-Thoraval F (2013) The burden of liver disease in Europe: a review of available epidemiological data. J Hepatol. 58:593-608.

2. Cadranel JF, Rufat P, Degos F (2000) Practices of liver biopsy in France: results of a prospective nationwide survey. For the Group of Epidemiology of the French Association for the Study of the Liver (AFEF). Hepatology 32:477-481.

3. Piscaglia F, Salvatore V, Mulazzani L, Cantisani V, Colecchia A, et al. (2017) Differences in liver stiffness values obtained with new ultrasound elastography machines and Fibroscan: A comparative study. Dig Liver Dis.

4. Shiina T, Nightingale KR, Palmeri ML, Hall TJ, Bamber JC, Barr RG, et al. (2015) WFUMB guidelines and recommendations for clinical use of ultrasound elastography: Part 1: basic principles and terminology. Ultrasound Med Biol 41:1126-47.

5. Ferraioli G, Filice C, Castera L, Choi BI, Sporea I, Wilson SR, et al. (2015) WFUMB guidelines and recommendations for clinical use of ultrasound elastography: Part 3: liver. Ultrasound Med Biol 41(5):1161-79.

6. Dietrich CF, Bamber J, Berzigotti A, Bota S, Cantisani V, et al. (2017) EFSUMB guidelines and Recommendations on the Clinical Use of Liver Ultrasound Elastography, Update 2017 (Long Version). Ultraschall Me 38:e16-e47.

7. Barr RG, Ferraioli G, Palmeri ML, Goodman ZD, Garcia-Tsao G, Rubin J, et al. (2015) Elastography Assessment of Liver Fibrosis: Society of Radiologists in Ultrasound Consensus Conference Statement. Radiology 276:845-61.

8. Goodman ZD (2007) Grading and staging systems for inflammation and fibrosis in chronic liver diseases. J Hepatol 47:598-607.
9. Bravo AA, Sheth SG, Chopra S (2001) Liver Biopsy. N Engl J Med 344:495-500.

10. Fraquelli M, Baccarin A, Casazza G, Conti CB, Giunta M, M, et al. (2016) Liver stiffness measurement reliability and main determinants of point shear-wave elastography in patients with chronic liver disease. Aliment Pharmacol Ther 44:356-65.

11. Orlacchio A, Bolacchi F, Antonicoli M, Coco I, Costanzo E, Tosti D (2012) Liver elasticity in NASH patients evaluated with real-time elastography (RTE). Ultrasound Med Biol 38:53744.

12. Toshima T, Shirabe K, Takeishi K, Motomura T, Mano Y, Uchiyama H (2011) A new method for assessing liver fibrosis based on acoustic radiation force impulse: a special reference to the difference between right and the left liver. J Gastroenterol 46:705-11.

13. (2015) EASL-ALEH Clinical Practice Guidelines: Noninvasive tests for evaluation of liver disease severity and prognosis. J Hepatol 63:237-64.

14. Ferraioli G, Tinelli C, Lissandrin R, Zicchetti M, Bertuzzi S (2014) Ultrasound point shear-wave elastography assessment of liver and spleen stiffness: effect of training on the repeatability of measurements. Eur Radiol 24: 1283-9.

15. Mancini M, Salomone Megna A, Ragucci M, De Luca M, et al. (2017) Reproducibility of shear wave elastography (SWE) in patients with chronic liver disease. PLoS One 12:e185391.

16. Suh CH, Kim SY, Kim KW, Lim YS, Lee SJ, Lee MG, et al. (2014) Determination of normal hepatic elasticity by using real-time shear-wave elastography. Radiology 271:895-900.

17. Bota S, Herkner H, Sporea I, Salzl P, Sirli R, et al. (2013) Meta-analysis: ARFI elastography versus transient elastography for the evaluation of liver fibrosis. Liver Int. 33:1138-47.

18. Piscaglia F, Salvatore V, Di Donato R, D'Onofrio M, Gualandi S, et al. (2011) Accuracy of VirtualTouch Acoustic Radiation Force Impulse (ARFI) imaging for the diagnosis of cirrhosis during liver ultrasonography. Ultraschall Med 32:167-75. 
19. Cooper C (2012) Hepatitis C treatment highlights from the 2011 American Association for the Study of Liver Disease meeting. Clin Infect Dis 55:418-425.

20. (2002) National Institutes of Health Consensus Development Conference Statement: Management of hepatitis C 2002 (June 10-12, 2002). Gastroenterology 123:2082-2099.

Submit your manuscript to a JScholar journal and benefit from:

ฯ Convenient online submission

๑ Rigorous peer review

9 Immediate publication on acceptance

ब Open access: articles freely available online

ब High visibility within the field

ब Better discount for your subsequent articles

Submit your manuscript at http://www.jscholaronline.org/submit-manuscript.php 